\title{
TABES DORSALIS
}

\section{WITH SPECIAL REFERENCE TO PRIMARY OPTIC ATROPHY*† BY

\author{
RICHARD D. HAHN
}

\begin{abstract}
From the Chronic Disease Division of the Medical Clinics, Johns Hopkins University and Hospital, Baltimore, Maryland
\end{abstract}

For many years, dating back to about 1930, neurosyphilis in general and primary optic atrophy of syphilitic origin in particular have been the subjects of special interest and repeated study in the Syphilis Division of the Department of Medicine and the Department of Ophthalmology of the Johns Hopkins Hospital. As to optic atrophy, Moore, Woods, and myself are responsible for the concepts and data upon which the following remarks are based.

Prevention of Neurosyphilis in General, and of Tabes and Optic Atrophy in Particular.-Clinical neurosyphilis has not yet been observed to develop following the penicillin treatment of early (primary or secondary) syphilis. Penicillin is of equal preventive value when given for so-called asymptomatic neurosyphilis manifested by abnormal spinal fluids, in the absence of signs or symptoms of neurosyphilis. (Hahn, Cutler, Curtis, and nine others, 1956).

We recently engaged in a cooperative study of 765 patients with asymptomatic neurosyphilis, 50 per cent. of whom were followed for 3 years, 25 per cent. for 5 years, and 20 per cent. for more than 5 years. As shown in Fig. 1, penicillin is remarkably effective in preventing clinical progression when given for asymptomatic neurosyphilis; only 3 per cent. of such patients had developed any objective evidence of neurosyphilis (in all cases minor) by the seventh year after treatment. For comparison, there is also shown the probability of developing clinical neurosyphilis among 467 patients with asymptomatic neurosyphilis treated predominantly by metal chemotherapy. In the penicillin group, progression had practically ceased after the third year of observation, but after metal chemotherapy it continued to increase with increasing observation periods. The recorded progressions among the 765 patients treated with penicillin were objective rather than subjective, never disabling, and frequently questionable. Neither tabes nor primary optic atrophy, nor

* Received for publication June 17, 1957.

+ Paper read at the National Hospital for Nervous Diseases, Queen Square, London, on May 16, 1957.

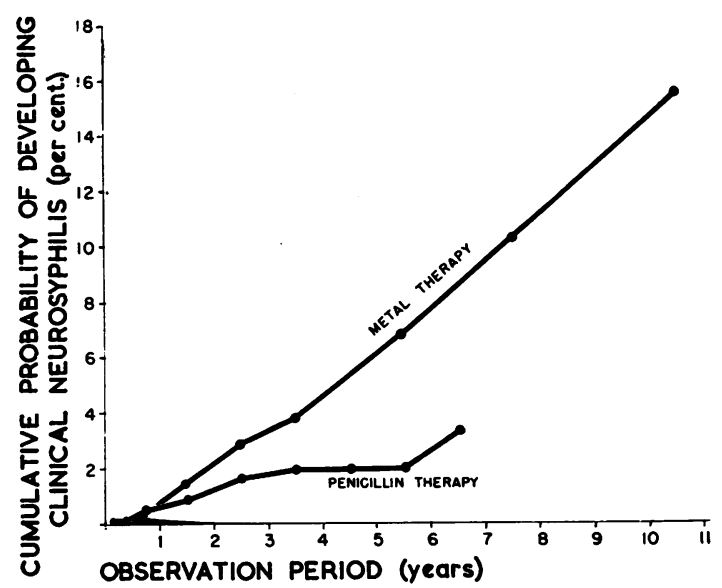

FIG. 1.-Asymptomatic neurosyphilis. The cumulative probability of developing clinical neurosyphilis among 765 patients after treatment with penicillin, and among 467 patients after treatment predominantly with metals (From Hahn and others, 1956).

indeed any form of parenchymatous neurosyphilis, was observed to develop.

Pathology of Primary Optic Atrophy.-This has been studied for many years, but there are still gaps in our knowledge. There is general agreement that the process begins in the intracranial portion of the optic nerve distal to the chiasma. The degeneration usually occurs first in the marginal fibres of the nerve, and there is thickening and perivascular round-cell infiltration of the overlying membranes, especially the pia, and of the connective tissue septa of the nerve. There is, however, no agreement as to the important question of the relationship, if any, of the inflammatory changes in the membranes to the degenerative process in the nerve itself, since the former are frequently found in neurosyphilitics who do not show the latter. The development of optic atrophy probably does not depend on the actual presence of Treponema pallidum in the optic nerve or visual pathways. Although the organism can be demonstrated in one of these locations in a very small 
proportion of patients with optic atrophy, it can likewise be so demonstrated in patients without optic atrophy.

In our material, primary optic atrophy occurs more than twice as frequently in tabes as in other types of neurosyphilis; in paresis uncomplicated by tabes, it is rare. Many patients show primary optic atrophy and pupillary changes as the only clinical manifestations of neurosyphilis (so-called "preataxic" optic atrophy). An analysis with respect to time of appearance after infection, rate of progression, and cerebrospinal fluid findings has failed to disclose any significant difference between this latter group and the group with clinical tabes. It is therefore probable that the fundamental pathological process is the same regardless of the extent and type of associated neurological findings. The presence of primary optic atrophy alone is sufficient to indicate parenchymal involvement, whether or not obvious tabetic posterior column damage is present. The frequent association with tabes dorsalis as well as the similarity of the optic nerve lesion to the posterior root lesion of tabes suggests that the pathogenesis of optic atrophy is identical with the pathogenesis of tabes dorsalis itself.

Dr. F. Ford (personal communication) has pointed out that the peculiar selectivity of tabes for the posterior roots of the spinal cord, pupillary fibres, optic nerve, and the sensory distribution of the second branch of the fifth nerve, may be more apparent than real. The one common characteristic of these lesions is that they involve that portion of the sensory fibres which lies between the sensory nerve cell and the central nervous system. This proximal portion of sensory fibres does not regenerate, presumably because of the lack of neurilemma sheath. This being the case, the ultimate clinical manifestations of tabes may be the end result of repeated minor lesions. Motor nerve involvement, in similar circumstances, might never reach a clinical threshhold because of the capacity of the axons of motor nerves for regeneration.

Pathogenesis.-The pathogenesis of optic atrophy, like that of tabes dorsalis, is unknown. Numerous theories have been propounded, none of which is entirely satisfactory. Any theory of the pathogenesis of the peculiar symptom complex of tabes and primary optic atrophy must take into consideration the fact that tabes differs in certain important respects from all other types of neurosyphilis:

(1) Its longer incubation period (24 years average in our material).

(2) The peculiar selectivity of tabes for the posterior roots and columns of the spinal cord; and its nearly complete limitation in the cerebrum to isolated disturbances, e.g. pupillary changes, disturbances of sensation in the distribution of the second branch of the fifth cranial nerve, oculomotor palsies, and primary optic atrophy. Diffuse meningovascular neurosyphilis and paresis exercise no such selectivity.

(3) The relative refractoriness of tabes to treatment.

(4) The relatively low antibody content of both blood and spinal fluid in tabes as compared with other types of neurosyphilis. With standard serological tests of average sensitivity, the blood test is negative in about 20 per cent. of tabetics and the spinal fluid complement-fixation test in an equal percentage. Those which are positive are often weakly so. This weak antibody response applies both to reagin and to antibodies specific for $T$. pallidum (the TPI test).

(5) Most important of all, the lesions of tabes are remarkable for the absence rather than the presence of $T$. pallidum. Though organisms have been sought by neuropathologists the world over, they have been found in only a few cases. Still more rarely has $T$. pallidum been found in the actual visual pathways of pure tabetics, most reports of positive findings being in patients with paresis or taboparesis.

(6) Most tabetics are of asthenic bodily habitus.

It is also clear that in rare instances the clinical picture of tabes may be found in individuals who give no history of syphilis, have negative blood and spinal fluid serologic findings (including a negative TPI test), and present other conditions suggesting a deficiency disease such as diabetes mellitus, chronic alcoholism, or pernicious anaemia. Indeed, it has long been recognized that tabes is prone to occur in ill-nourished individuals, though it is not clear whether the malnutrition precedes or succeeds the tabes. Spinal cord lesions resembling those of tabes have been produced in experimental animals by diets deficient in vitamin $A$ in some cases, and in pyridoxine and pantothenic acid in others.

These facts have led to the hypothesis that syphilis is perhaps not the sole cause of tabes dorsalis and of primary optic atrophy, but that there may be an associated, and as yet unknown, $\mathbf{X}$ factor, perhaps nutritional.

The theory ascribing syphilitic primary optic atrophy to adhesive optochiasmatic arachnoiditis is no longer widely held. The existing evidence indicates that the occurrence of such adhesions about the chiasma and optic nerves is largely coincidental, and is certainly not the explanation for the usual primary optic atrophy of tabes. Those few 
patients of ours who have been surgically investigated have not shown such adhesions.

Clinical Picture.-This is so well known that it requires but brief mention, although it should be emphasized that, on ophthalmoscopic examination alone, it is impossible to differentiate the atrophy of central nervous system syphilis from that caused by certain brain tumours, intracranial aneurysms, arteriosclerosis, often disseminated sclerosis, and sometimes toxic amblyopias, and occasionally from the optic atrophy of glaucoma and thrombosis of the central retinal artery.

Pupillary changes are, however, almost constant in the syphilitic type. The media are clear, the disks normally being outlined with preservation of the physiologic cups. There is a gradually developing bluish-white pallor of the nerve, which usually involves the whole of it, sometimes with accentuation of atrophy in the papillo-macular bundle. There are no associated retinal or choroidal lesions and, if such are present, they are only coincidental. In advanced cases, there is some constriction of the arteries, probably due to the secondary fibrosis of the nerve itself with resulting traction on the central vessels. The end picture is advanced generalized pallor of the nerve without visible ophthalmoscopic evidence of active or old inflammation.

The failure of visual acuity may occur early or late according to whether there is early degeneration of the papillo-macular bundle, or degeneration beginning in the peripheral fibres and slowly progressing centrally (Fig. 2).

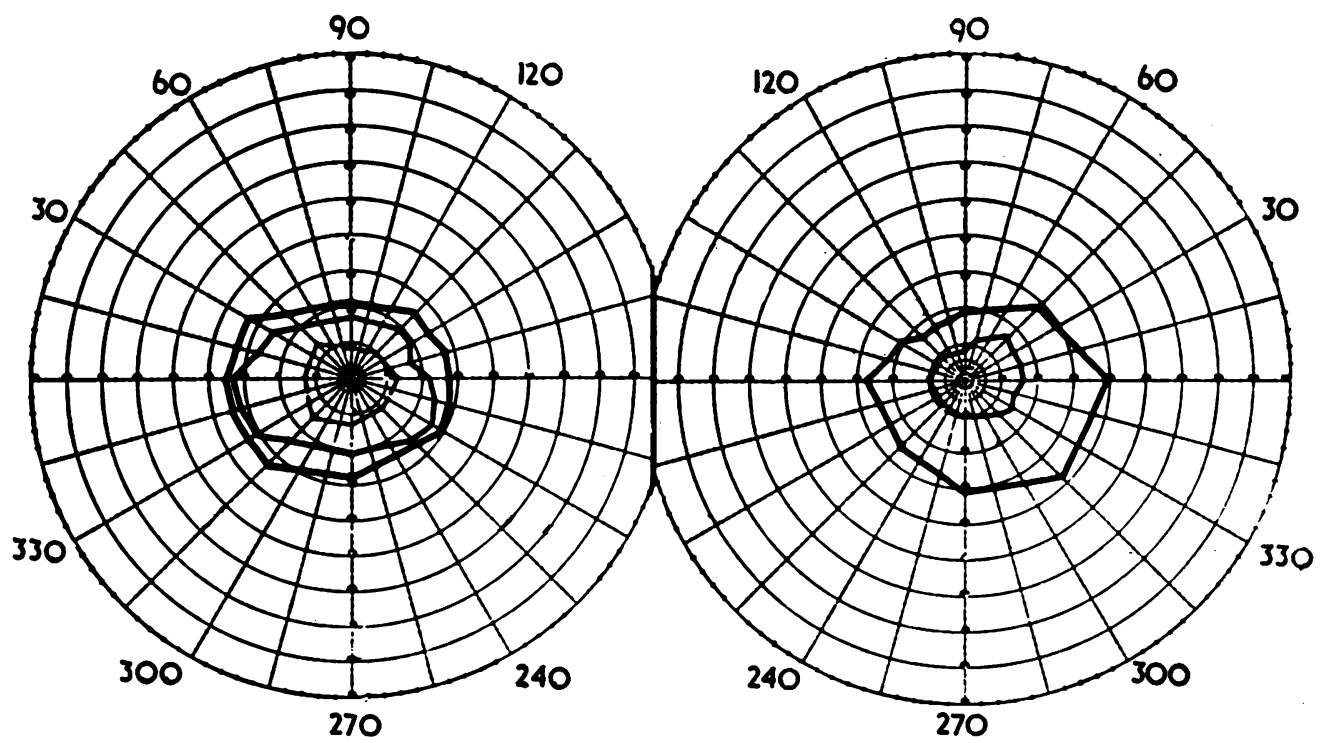

FIG. 3. - Concentric contraction of the visual fields in syphilitic primary optic atrophy (From Sloan and Woods, 1938).

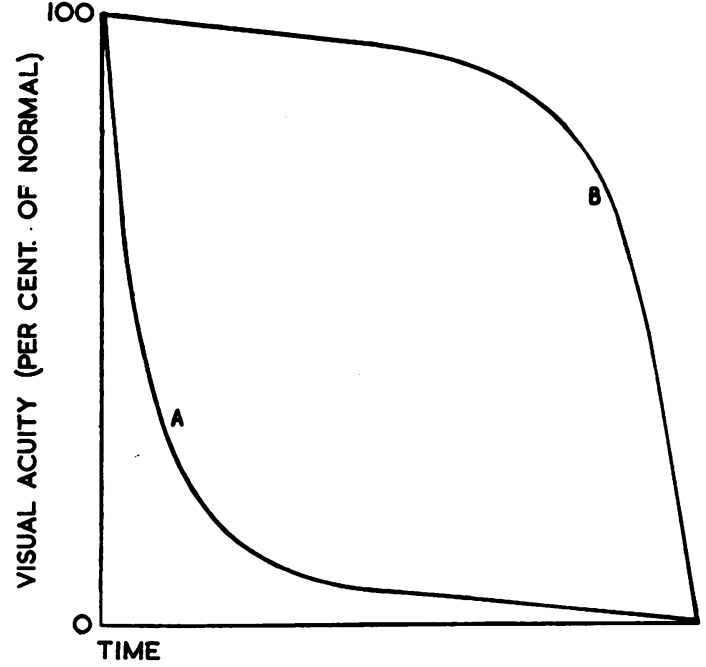

Fig. 2.-Rate of loss of central visual acuity in (A) patients with central or cecocentral scotoma and (B) patients with peripheral constriction of the visual fields only (From Woods, 1943).

Perimetric studies by Sloan and Woods (1938) involving repeated visual fields in 56 patients with syphilitic primary optic atrophy throughout the course of their disease, indicate that the field defects are of four separate types:

(1) Concentric contraction of the visual fields associated with late loss of visual acuity. This occurred in 12 per cent. of their cases (Fig. 3). 


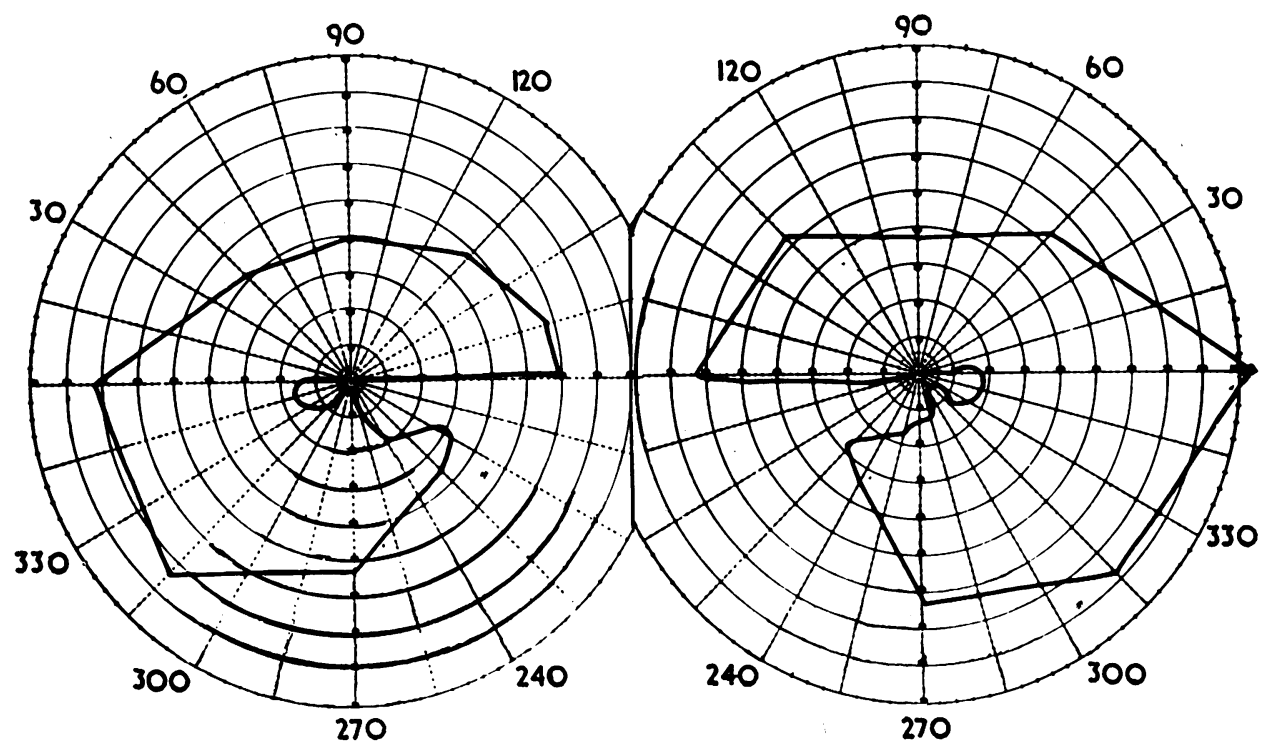

FIG. 4.-Localized sector-like defects in the visual fields in syphilitic primary optic atrophy (From Sloan and Woods, 1938).

(2) Sector-shaped or nerve bundle defects, with loss of vision which may be early or late according to the degree of involvement of the papillo-macular bundle. This was present in 34 per cent. of the patients examined (Fig. 4).

(3) Central or cecocentral scotomata with normal peripheral fields associated with early loss of vision. Fourteen per cent. of the cases showed this type of defect (Fig. 5).

(4) Central or cecocentral scotomata with defects in the peripheral fields associated with early loss of vision, occurring in 40 per cent. of the cases (Fig. 6, opposite).

The incidence of central scotomata and early loss of useful vision (53 per cent. of all the cases examined) is in striking contrast with the old idea of the comparative infrequency of central scotomata and of late loss of vision.

In patients with the peripheral type of constriction without early visual failure, defects in the visual fields may precede not only any loss of vision, but also any ophthalmoscopic evidence of pallor or atrophy of the nerve. In tabetics, a demonstrated and confirmed visual field defect for which there is no adequate explanation may indicate impending degeneration of the nerve. In the early diagnosis of syphilitic optic nerve disease, therefore, careful perimetry is essential.

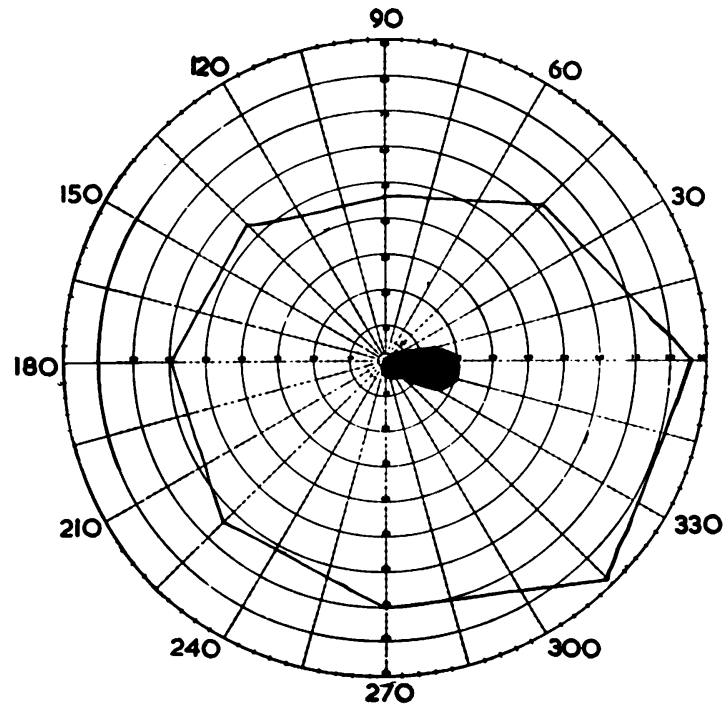

Fig. 5.-Central scotoma with a normal peripheral field in syphilitic primary optic atrophy (From Sloan and Woods, 1938).

Differential Diagnosis. - The knowledge that syphilis is by far the commonest aetiologic agent is of considerable value. In order to determine the cause, a painstaking general study of the patient should include, in addition to careful perimetry, a complete neurological and physical examination, $x$ rays of the skull, a serological test for syphilis, 


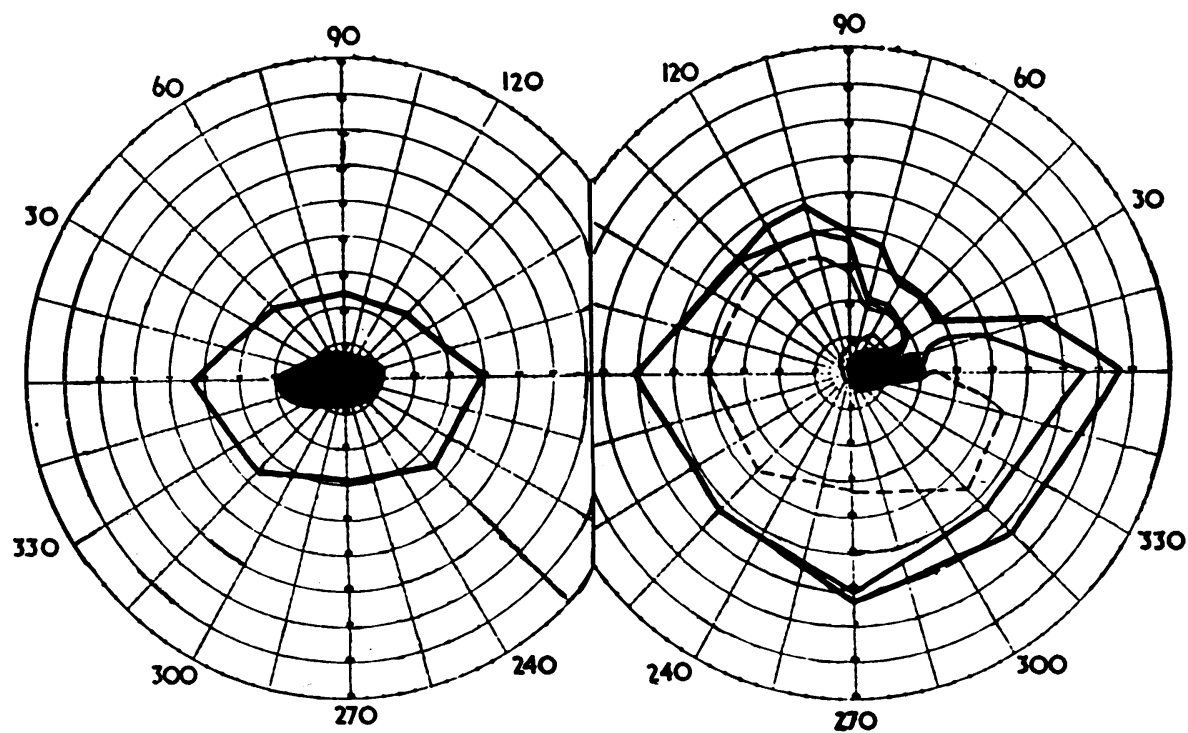

FIG. 6.-Central scotoma with peripheral field defect in syphilitic primary optic atrophy (From Sloan and Woods, 1938).

and an examination of the cerebrospinal fluid. The presence of pupillary changes, signs of posterior column damage, even though minor, and an abnormal cerebrospinal fluid are of particular value in pointing to a syphilitic aetiology. In only 7 per cent. of 221 patients with syphilitic primary optic atrophy was the spinal fluid entirely normal. Very unusual in syphilitic primary optic atrophy are normal pupils, bitemporal or binasal hemianopsia, and central scotomata with normal peripheral fields, although the latter do occur.

The importance of syphilis in optic nerve disease has been shown in three studies from the Department of Ophthalmology of the Johns Hopkins Hospital. There were 129 cases of primary optic atrophy in these three studies, of which 66 (51 per cent.) were due to syphilis of the central nervous system.

Looked at from another point of view, in my own clinic, primary optic atrophy was found to occur in approximately 8 per cent. of all patients with clinical evidence of acquired neurosyphilis. The incidence was higher in the neurosyphilitic Negro than in the neurosyphilitic white patient. It occurred more than twice as frequently in tabes as in other types of neurosyphilis. In uncomplicated paresis it was found to be rare, although it was common in taboparesis. Many patients, however, presented primary optic atrophy and pupillary changes as the only clinical manifestations of neurosyphilis.
Results of Treatment.-With regard to the effects of therapy upon tabetic symptoms other than primary optic atrophy, the physical signs among 101 tabetic patients rarely progressed and practically never disappeared after treatment with penicillin alone or with penicillin plus malaria. As illustrated in the Table, mild to moderate tabetic symptoms disappeared in one-third of 208 instances with penicillin alone, and in one-third of 67 instances with penicillin plus malaria. Severe symptoms rarely disappeared with penicillin alone or with penicillin plus malaria.

\section{TABLE*}

OUTCOME OF TABETIC SYMPTOMS AFTER TREATMENT WITH PENICILLIN OR WITH PENICILLIN PLUS MALARIA. (MEDIAN FOLLOW-UP FOR 18 MONTHS)

\begin{tabular}{l|c|c}
\hline Treatment & $\begin{array}{c}\text { Disappearance of } \\
\text { Mild to Moderate } \\
\text { Symptoms } \\
\text { (Instances) }\end{array}$ & $\begin{array}{c}\text { Disappearance of } \\
\text { Severe Symptoms } \\
\text { (Instances) }\end{array}$ \\
\hline Penicillin alone 달 & $\frac{1}{3}$ of 208 & 1 out of 27 \\
\hline Penicillin plus Malaria & $\frac{1}{3}$ of 67 & 1 out of 7 \\
\hline
\end{tabular}

The effect of penicillin dosage and duration of treatment upon tabetic symptoms has not yet been completely evaluated. Since the median period of clinical follow-up in our patients was only about 18 months, no conclusions could be drawn as to the permanence of the results. That penicillin alone can

\footnotetext{
* Adapted from Hahn and others, 1951.
} 
occasionally alleviate mild to moderate tabetic symptoms over a long period of time is, however, strongly suggested by the course of a small group of patients followed more than 3 years after treatment. Complete relief of paraesthesiae was seen in eight patients, of lightning pains in six, of ataxia in three, and of bladder symptoms in eight, and in five patients libido and potentia returned to normal for their ages.

In a few patients, certain symptoms developed or became worse after therapy. This was particularly true of lightning pains and bladder symptoms. It is probable that tabetics with pleocytosis in the cerebrospinal fluid at the time of treatment may be expected to have, on the whole, better results, although no absolute correlation was statistically evident in our limited material.

It is of interest that, in our tabetic patients, the Herxheimer reaction after penicillin was infrequent and limited to a febrile reaction without obvious adverse clinical effect. This is in marked contrast to the frequency and severity of this reaction in paresis and in acute syphilitic meningitis, where large numbers of $T$. pallidum are present in the nervous system. The minor Herxheimer response is in keeping with the paucity of $T$. pallida in the nerve tissues of tabetics.

The rate of progression of untreated optic atrophy, as estimated from historical data, is extremely variable. The extremes of variability are illustrated by 33 of our own patients who became blind less than 6 months after the onset of symptoms, and by fourteen patients who had not become blind 5 or more years after the onset of symptoms.

We have studied the effect of treatment upon the course of tabetic primary optic atrophy over a period of almost two decades. In 1942 we believed that we had demonstrated the superior value of malaria therapy in a selected group of slow progressors. Even this limited conclusion, however, is not statistically valid for various reasons, chiefly because of the absence of a comparable group of untreated control patients. For the same reason, the more recent literature is even less satisfactory. To estimate the efficacy of therapeutic procedures in any chronic variable disease, one must know accurately the prognosis in the absence of treatment. In the case of syphilitic optic atrophy this requirement has not been wholly met. Few patients in our previous study, or in any other so far reported, have remained untreated after initial observation. The course of untreated optic atrophy had, of necessity, to be estimated from anamnestic historical data which were influenced by a multiplicity of extraneous factors and derived largely from a group of patients already blind when first seen. Using this method, we found in 1942 that blindness, defined as visual acuity of $10 / 200$ or less in the better eye, occurred in 28 per cent. of patients within 1 year of the onset of symptoms, in 50 per cent. within 2 years, in 65 per cent. within 3 years, and in 75 per cent. within 6 years (Fig. 7). It is unlikely that any significant alteration in these figures, based upon 252 person-years of retrospective analysis, will result from any further study.

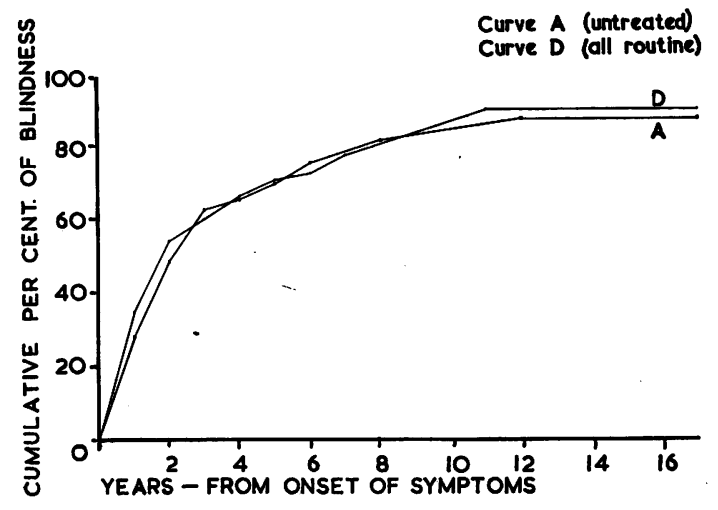

Fig. 7.-Cumulative probability of blindness related to years after onset of symptoms in untreated (Curve A) and metal-treated (Curve D) syphilitic primary optic atrophy (From Moore and others, 1942).

Nevertheless, it is manifestly not a sound device to compare the observed course after treatment with historical data concerning the estimated course before observation and treatment, since the two sets of data are not comparable. The former is objective, while the latter is subjective and is influenced by a multiplicity of extraneous factors, including the care with which the history is taken, the accuracy of the patient's memory, unilateral onset in which one eye may become completely blind before the other is significantly involved (hence the patient may be unaware that he has only monocular vision), and the type of visual-field defect. A central scotoma obviously precipitates subjective symptoms of visual failure earlier than a slowly developing concentric constriction of the visual fields. In the analyses of individual case records, it was repeatedly noted that the elicited date of onset of symptoms varied from examiner to examiner.

For purposes of objectivity, in our most recent study (Hahn, Zellmann, Naquin, Cross, and Marcus, 1955) the course of the disease was considered to date from the time of the initial Johns Hopkins Hospital observation rather than from the time of onset of symptoms. The life-table method 
was utilized to describe this course between the initial and the latest observation.

Only patients with visual acuity greater than $10 / 200$ at the time of our initial observation were included, thus eliminating the fallacy of the classification of the non-progression of an industrially blind patient as a treatment success, as is so often claimed elsewhere in the literature.

The percentage of visual efficiency was determined for each eye, using the visual acuity, corrected whenever available, and the conversion table as recommended by the report of the Committee on Compensation for Eye Injuries of the American Medical Association. Visual efficiency for both eyes was determined by adding together the visual efficiency for each eye and dividing the result by two. Results are expressed in terms of the percentage loss of pre-treatment visual efficiency.

Because patients were often subjected to multiple types of treatment, it was not possible to consider each form of therapy separately. Treatment groups, based solely upon therapy given after the initial Johns Hopkins Hospital observation, are as follows:

(1) No treatment after initial observation at the Johns Hopkins Hospital (but with heterogeneous treatment elsewhere previously).

(2) Metal chemotherapy (with or without subdural therapy).

(3) Malaria without penicillin (with or without metal and subdural therapy).

(4) Penicillin and malaria.

(5) Penicillin without malaria.

The "no-treatment" group was in fact a heterogeneous one, in which the patients had largely been treated elsewhere by various methods; thus it did not constitute a true untreated control group. The other groups were largely mutually exclusive, even considering previous treatment before our first observation.

Comparison of different treatment methods is best made when they are as nearly concurrent in time as possible. The present material falls far short of this ideal. The patients in Group 2 (metal chemotherapy) were all first observed between 1920 and 1943. The majority of those in Group 3 (malaria without penicillin) also belonged to this period, the remainder being scattered up to 1948 . Group 4 (malaria plus penicillin) and Group 5 (penicillin alone) were, of course, first observed from 1943 onwards.

The treatment groups were similar with respect to neurological diagnosis, degree of pre-treatment visual field constriction, percentage of inactive spinal fluids with normal cell count and protein, and apparent rate of progression as judged by scatter charts before our first treatment.

However, they were not strictly comparable in respect of age. Those patients given malaria were predominantly younger than those patients treated by other methods. With regard to race, there were striking dissimilarities between the basic treatment groups. Malaria without penicillin was given in a ratio of almost $3: 1$ to whites; penicillin without malaria was given in a ratio of over $3: 1$ to Negroes; in the other treatment groups the ratio approached 1 : 1. Another difference of considerable importance is that, by and large, those patients given penicillin alone had the symptoms longer and had somewhat lower visual efficiency on admission to the study than did patients in the other treatment groups.

Another and extremely important difference between these groups was in the percentage of patients in each who returned for follow-up. The "treated elsewhere, no treatment at Johns Hopkins Hospital" group were so poorly followed as to preclude any attempt at determining the results. The percentage of patients followed was poor for the metal-treated group and relatively good for the more recently treated malaria, malaria plus penicillin, and penicillin groups. This difference in degree of follow-up introduces yet another serious bias in comparing the treatment groups, particularly those treated with metals and those given penicillin, malaria, or both. Since only 56 per cent. of the metal-treated group returned for second observation, any such comparison necessitates the large assumption that the results in the 44 per cent. who were not followed were similar to the 56 per cent. who were followed.

Despite these many differences in basic data between the treatment groups, we have thought it worth while to determine the outcome in order to present the picture of the rate of progression of primary optic atrophy under actual observation after treatment, and because, in spite of its many deficiencies, the present material is the best available in the literature thus far. Moreover, in view of the decreasing incidence of syphilis, it seems unlikely that better material will become available in the foreseeable future.

Fig. 8 (overleaf) shows the cumulative probability of losing 10 per cent. or more of initial visual efficiency according to the type of treatment received. The actual life-tables upon which this and subsequent figures are based are not shown, but, in general, calculations were terminated when only one-third of the patients remained under observation. 


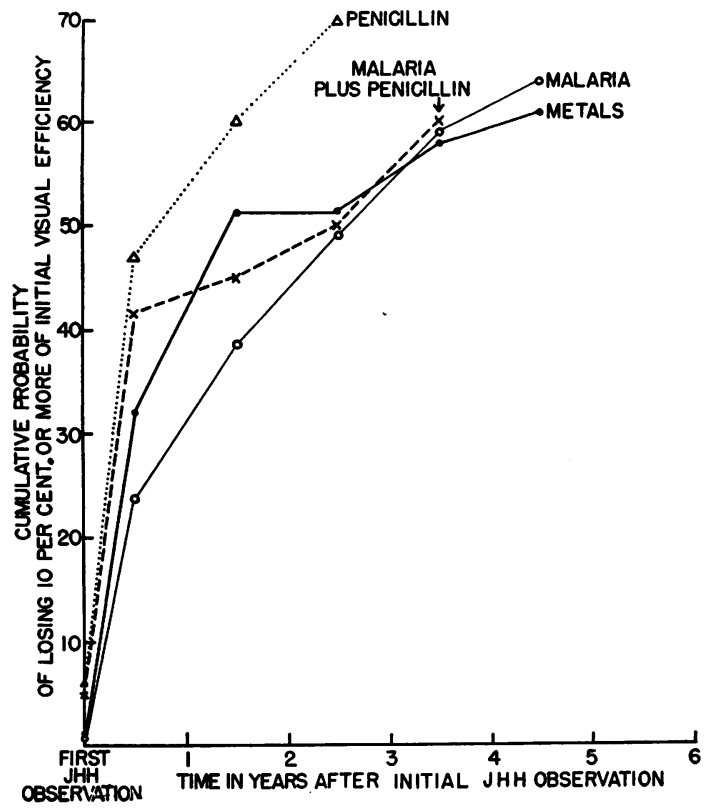

FIG. 8.-Cumulative probability of losing 10 per cent. or more of initial visual efficiency, according to type of treatment received (From Hahn and others, 1955).

There are no striking differences in outcome between the basic treatment groups. The one conclusion which may be drawn is that in a high proportion of cases no form of treatment seems able to arrest progression. In the various treatment groups, the rate of progression 3 years after treatment was from 50 to 70 per cent. of the patients

Fig. 9 shows the cumulative probability of losing 80 per cent. or more of initial visual efficiency according to the type of treatment received. Again the differences between treatment groups do not appear to be significant. Fig. 9 is of interest, however, in that it gives an entirely different picture of the prognosis of syphilitic optic atrophy under direct observation as compared with that derived from questioning already blind patients about the duration of their symptoms (cf. Fig. 7). Moreover, the configuration of the curves, with their lack of late rise and their flattening out after the first few years, suggests that, with treatment, the prognosis for prolonged retention of some useful vision, even at a low level, is relatively good. The patients treated with metals or with malaria alone were followed the longest, and even 10 to 11 years after treatment, only 21 and 13 per cent. respectively had lost 80 per cent. of initial visual efficiency.

The variable course of syphilitic primary optic atrophy suggests the possible influence of factors

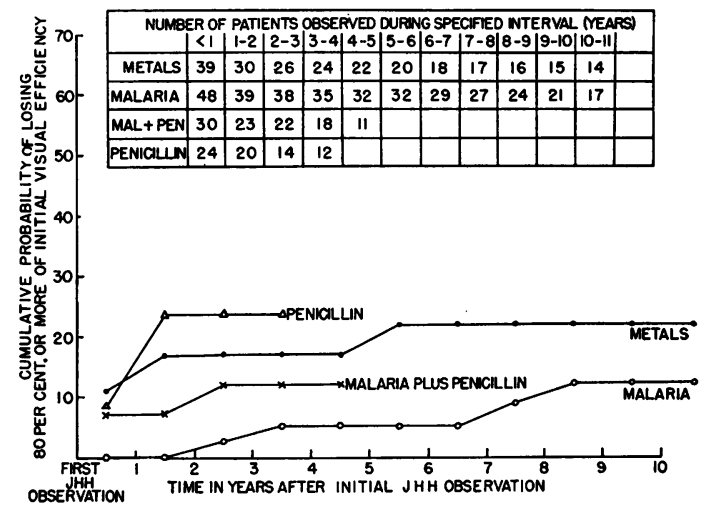

Fig. 9.-Cumulative probability of losing 80 per cent. or more of initial visual efficiency, according to type of treatment received (From Hahn and others, 1955).

other than the type of treatment administered in determining the outcome. In general, neurosyphilis with an active cerebrospinal fluid (with elevated cell count and protein) responds better to treatment than does neurosyphilis with an inactive spinal fluid. This is an obvious sign of the "curability" of the inflammatory as compared with the degenerative component of the lesions. In primary optic atrophy, however, the rate of progression after treatment was not apparently significantly influenced by the spinal fluid cell count (Figs 10 and 11, opposite).

In order to evaluate the more or less generally held clinical impression that treatment administered while vision is still relatively good is more effective than treatment given after vision has greatly deteriorated, our patients were divided into two groups irrespective of the type of treatment administered. Two or more observations were available for 93 patients with initial visual efficiency of 40 per cent. or more and for 65 patients with initial visual efficiency less than 40 per cent. The former did significantly better than the latter, progression occurring at 4 to 5 years in 40 per cent. and 66 per cent. respectively (Fig. 12, opposite).

Likewise, a loss of 80 per cent. or more of initial visual efficiency, even as long as 10 to 11 years after the first observation, occurred in only 9 per cent. of those with relatively good visual efficiency as compared with 26 per cent. of those with more advanced visual failure (Fig. 13, opposite).

In order to present a composite picture of the course of treated syphilitic primary optic atrophy, there is shown the cumulative probability of losing 10 per cent. or more and also 80 per cent. or more of initial visual efficiency in 141 patients after various types of treatment (Fig. 14, see p. 148). This graph is considered accurately to portray the course 


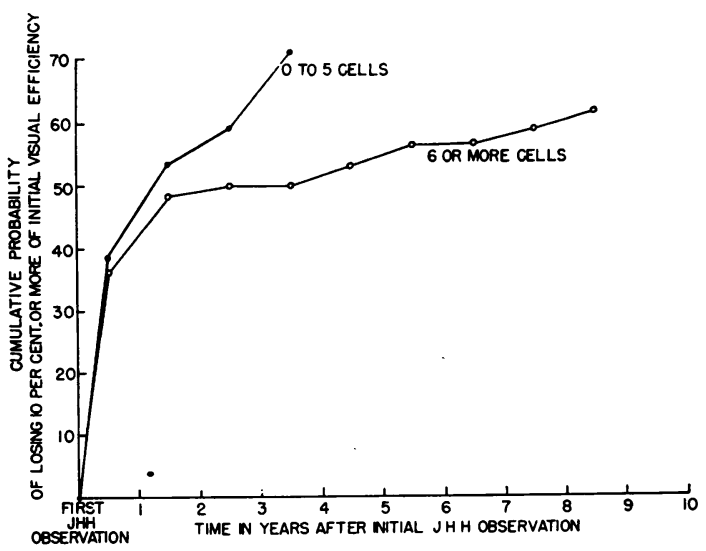

\begin{tabular}{|c|c|c|c|c|c|c|c|c|c|c|c|}
\hline NUMBER OF PATENTS OBSERVE DURING SPECIFIED INTERVAL MEARS) \\
\hline & $<1$ & $1-2$ & $2-3$ & $3-4$ & $4-5$ & $5-6$ & $6-7$ & $7-8$ & $8-9$ & \\
\hline O TO5 CELLS & 49 & 26 & 17 & 15 & & & & & & \\
\hline $\begin{array}{c}\text { 6 OR MORE } \\
\text { CELLS }\end{array}$ & 70 & 41 & 32 & 31 & 28 & 24 & 22 & 21 & 20 & \\
\hline
\end{tabular}

FIG. 10.-Cumulative probability of losing 10 per cent. or more of initial visual efficiency by initial spinal fluid cell count (From Hahn and others, 1955).

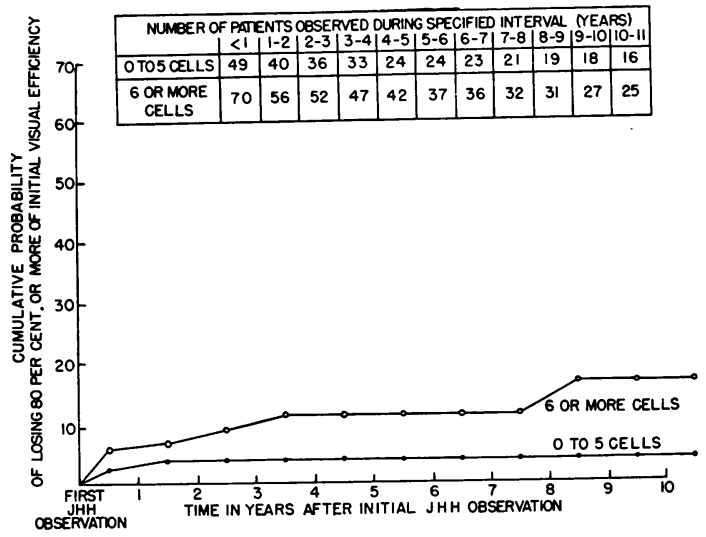

FIG. 11.-Cumulative probability of losing 80 per cent. or more of initial visual efficiency by initial spinal fluid cell count (From Hahn and others, 1955).

of treated syphilitic primary optic atrophy. The upper curve shows the cumulative probability of losing 10 per cent. visual efficiency. Here, there is a sharp initial progression in about one-third of the patients during the first year of post-treatment observation. Thereafter, the slope of the curve is more gradual, approximately one-half of the patients progressing by 2 to 3 years and two-thirds by 4 to 5 years.

The lower curve represents a loss of 80 per cent. of initial visual efficiency. On this basis, the longterm outlook seems better. The slope of the curve is gradual from the onset, and increases slowly and
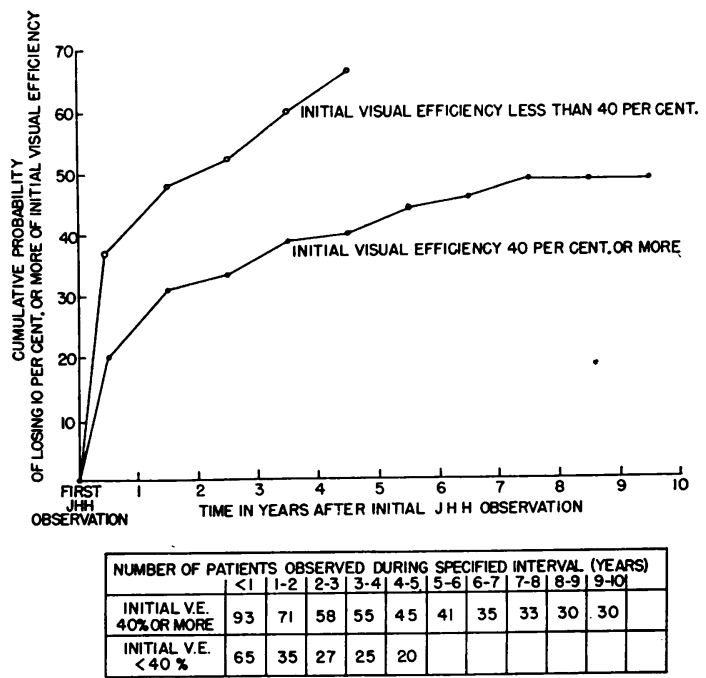

FIG. 12.-Cumulative probability of losing 10 per cent. or more of initial visual efficiency by initial visual efficiency (From Hahn and others, 1955).

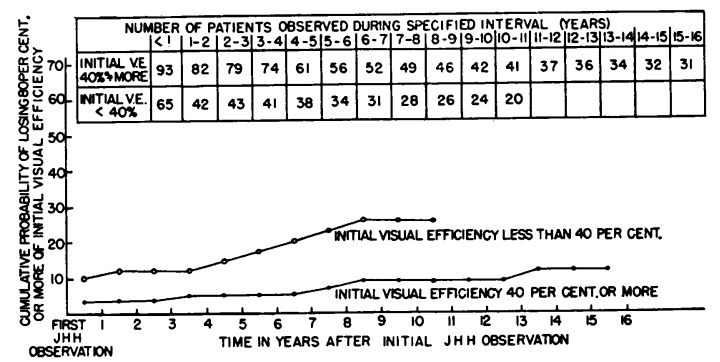

FIG. 13.-Cumulative probability of losing 80 per cent. or more of initial visual efficiency by initial visual efficiency (From Hahn and others, 1955).

at a relatively uniform rate throughout the observation period. By 1 to 2 years, only 10 per cent. of patients have attained this degree of progression, by 4 to 5 years only 15 per cent., and by 7 to 8 years only 20 per cent.

\section{Conclusions}

We must then conclude that our material, like all previous and later studies, does not permit a valid comparison between the effects of various types of treatment in syphilitic primary optic atrophy. Deficiencies in basic data preclude accurate comparisons, and such differences in outcome as there may be after various types of treatment are perhaps obscured by other variables. It does seem, however, 


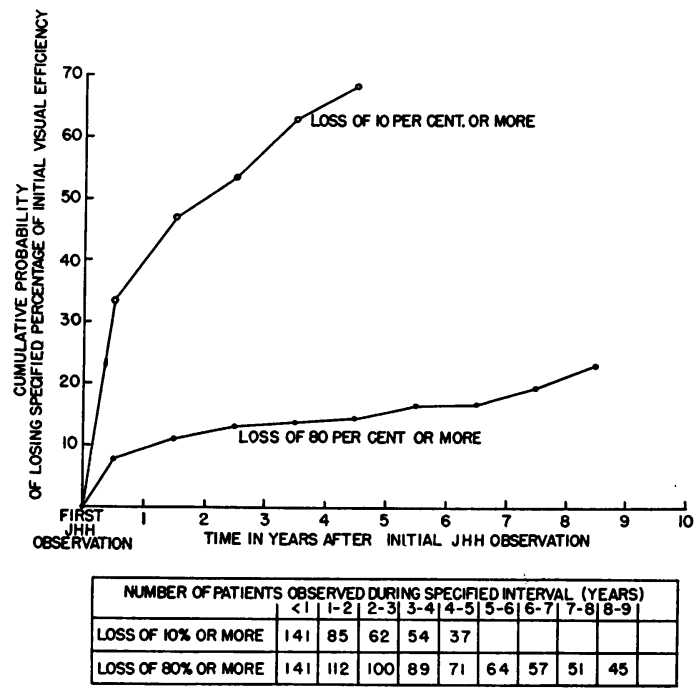

FIG. 14.-Cumulative probability of losing specified percentage of initial visual efficiency in 141 patients, after various types of treatment (From Hahn and others, 1953).

that the rate of progression after treatment is not directly dependent upon the disease activity of the cerebrospinal fluid before treatment. It also seems clear that patients with a visual efficiency of more than 40 per cent. who are treated early show a significantly slower rate of progression after treatment than do patients with a lower visual efficiency who are treated later in the course of the disease. In our opinion, this difference may be attributed largely to the treatment given. The prognosis in treated optic atrophy for retention of some vision (i.e. for loss of less than 80 per cent. of visual efficiency) is relatively good; indeed, over a period of 7 to 8 years of direct observation after treatment, the visual acuity had deteriorated to this stage in only one patient in five.

\section{REFERENCES}

Hahn, R D Cutler, J. C Curtis A C Gammon G, Heyman, A. Johnwick, E, Stokes, J. H, Solomon, H. Thomas, E., TimberJohnwick, E., Stokes, J. H., Solomon, H., Thomas, E., Timberlake, W., Webster, 1 .

Arch. Derm., 74, 355 .
Lewis, B. I., Wiggall, R. H., Cross, E. S. Jr. (1951). Amer. J. Syph., 35, 433 .

Zellmann, H. E., Naquin, H., Cross, E. S. Jr., and Marcus, D. M. (1955). J. chron. Dis., 1, 601

Moore, J. E., Hahn, R. D. Woods, A. C., and Sloan, L. (1942). Amer. J. syph., 26, 407.

Sloan, L. L., and Woods, A. C. (1938). Arch. Ophthal. (Chicago),

Woods, A. C. (1943). Amer. J. Syph., 27, 133.

Additional Bibliography

Moore, J. E. (1932). Medicine (Baltimore), 11, 263. and Woods, A. C. (1940). Ibid., 24, 59. 